山्यRANÇAISE

$>\mathrm{DE}$

$\stackrel{1=1}{\simeq}$ PÉDAGOGIE
Revue française de pédagogie

Recherches en éducation

$181 \mid 2012$

Varia

\title{
Pourquoi les enseignants quittent-ils leur école? Revue de la littérature scientifique relative au turnover des enseignants
}

Why do teachers leave their schools? Review of the scientific literature relative to teacher turnover

Sandrine Lothaire, Xavier Dumay et Vincent Dupriez

\section{OpenEdition}

Journals

Édition électronique

URL : http://journals.openedition.org/rfp/3931

DOI : 10.4000/rfp.3931

ISSN : 2105-2913

Éditeur

ENS Éditions

Édition imprimée

Date de publication : 31 décembre 2012

Pagination : 99-126

ISBN : 978-2-84788-405-0

ISSN : 0556-7807

\section{Référence électronique}

Sandrine Lothaire, Xavier Dumay et Vincent Dupriez, « Pourquoi les enseignants quittent-ils leur école ? Revue de la littérature scientifique relative au turnover des enseignants ", Revue française de pédagogie [En ligne], 181 | 2012, mis en ligne le 17 juin 2015, consulté le 25 juillet 2020. URL : http:// journals.openedition.org/rfp/3931; DOI : https://doi.org/10.4000/rfp.3931 


\section{NOTE DE SYNTHÈSE}

\section{Pourquoi les enseignants quittent-ils leur école? Revue de la littérature scientifique relative au turnover des enseignants Sandrine Lothaire, Xavier Dumay et Vincent Dupriez}

Cette note de synthèse vise à appréhender pourquoi certains enseignants souhaitent ou décident de quitter leurlieu de travail, que ce soit pour se réorienter professionnellement ou pour exercer leur métier dans un autre établissement. Elle centre donc l'analyse de la mobilité professionnelle enseignante sur les établissements, en essayant de comprendre pourquoi certains sont plus exposés que d'autres au turnover des enseignants. Elle montre qu'au niveau individuel, des variables objectives telles que l'ancienneté, la formation initiale, la discipline enseignée et les caractéristiques sociodémographiques des enseignants influencent les taux de turnover, mais aussi que des facteurs individuels plus subjectifs comme la satisfaction professionnelle et l'engagement organisationnel représentent des éléments favorables au maintien du personnel dans la profession et dans l'établissement. Enfin, elle fait apparaître que la composition sociale, ethnique et académique des établissements ainsi que leurs caractéristiques organisationnelles sont également liées au turnover.

Mots-clés (TESE) : enseignant, établissement d'enseignement, mobilité des enseignants, mobilité professionnelle, système éducatif.

\section{INTRODUCTION}

Dans un grand nombre de pays, des études se sont penchées sur la mobilité professionnelle qui caractérise aujourd'hui le métier d'enseignant et, en particulier, les enseignants qui débutent dans la profession. Ainsi, aux États-Unis, il apparaît que $11 \%$ des enseignants quittent la profession en cours ou à l'issue de la première année d'exercice du métier et $39 \%$ dans les cinq ans (Ingersoll, 2002). Une étude menée dans l'enseignement secondaire en Belgique francophone (Vandenberghe, 
2000) fait également apparaître que quatre enseignants sur dix ont abandonné le métier au cours de leurs cinq premières années de carrière. Ces données sont le plus souvent présentées avec pour toile de fond des systèmes éducatifs qui rencontrent par ailleurs des difficultés à attirer de nouveaux enseignants (OCDE, 2001) et qui doivent faire face, comme c'est notamment le cas dans de nombreux États américains et dans plusieurs pays d'Europe (Malet, 2011 ; European Commission, 2012), à des situations de pénurie ou du moins de déficit d'individus titulaires du diplôme requis.

Ces résultats interpellent par rapport à une analyse des trajectoires professionnelles des enseignants et révèlent probablement une perte d'attractivité globale de la profession. Ce phénomène n'est pas neuf et de nombreux auteurs ont suggéré de le lire, entre autres, comme une conséquence de la désinstitutionnalisation de l'école (Dubet, 2002), accompagnée d'un « désenchantement des mythes qui sont le support de la vocation " (Demailly, 1991). D'autres auteurs ont traité cette question à travers le prisme de l'identité professionnelle des enseignants, de la complexité croissante du métier ou encore de son attractivité relative par rapport à d'autres professions (Maroy \& Cattonar, 2002 ; Rayou \& van Zanten, 2004).

De telles analyses sont évidemment pertinentes et requièrent un approfondissement destiné à mieux comprendre quelles sont les représentations sociales relatives au métier et les facteurs, tant objectifs que subjectifs, qui, aujourd'hui, freinent ou incitent les jeunes diplômés à rentrer et à persister dans la profession. Toutefois, elles n'offrent qu'une appréciation générale de la situation sur l'ensemble d'un territoire, qui est le plus souvent celui d'un système éducatif. Une ligne de recherche développée depuis une ou deux décennies tend pourtant à montrer que le phénomène relatif à la mobilité des enseignants mérite d'être étudié à d'autres échelles, et, en particulier, en relation avec les établissements scolaires où ceux-ci exercent.

En effet, si, de manière générale, la profession enseignante souffre d'une perte d'attractivité dans la plupart des régions du monde, il apparaît que les établissements d'enseignement sont inégaux dans leur capacité à attirer et à stabiliser leur personnel. C'est donc une littérature spécifique qui s'est développée autour de cette question de la rétention (locale) des enseignants et de l'analyse du turnover que vivent, parfois intensivement, certaines écoles qui rencontrent des difficultés pour stabiliser les membres de leur personnel. Globalement, ce sont le plus souvent celles situées en zone urbaine défavorisée (Lankford, Loeb \& Wyckoff, 2002 ; Smith \& Ingersoll, 2004) qui peinent davantage à attirer des enseignants et, ensuite, à les stabiliser en leur sein. Cette situation engendre, bien entendu, un ensemble de conséquences en entravant le développement d'un projet pédagogique propre à l'établissement ou la plausibilité d'un travail collectif entre enseignants construit dans la durée.

Toutefois, les recherches qui ont alimenté cette note de synthèse ${ }^{1}$ attirent l'attention sur le caractère beaucoup plus complexe de ce phénomène. En effet, tous les établissements scolaires accueillant un public défavorisé ne rencontrent pas des problèmes de stabilisation des enseignants et tous les enseignants ne sont pas attirés par le même type d'écoles. Ainsi, nous montrerons que les caractéristiques organisationnelles de l'établissement d'enseignement telles que le type de leadership, le climat et la solidarité entre enseignants ont également une influence significative sur la capacité des établissements à " garder " leurs enseignants.

Cette ligne de recherche a donc pour objectif prioritaire de comprendre pourquoi certains enseignants souhaitent ou décident de changer de lieu de travail. Elle conjugue, dans le déploiement de cette analyse, deux entrées complémentaires : d'une part, un examen des facteurs individuels objectifs (ancienneté, formation, discipline enseignée, etc.) et subjectifs (satisfaction professionnelle et engagement organisationnel) rendant certains individus davantage susceptibles de bifurquer professionnellement et, d'autre 
part, une analyse des organisations (le plus souvent, les établissements scolaires) dont la capacité à stabiliser leur personnel apparaît comme inégale.

Cette prise en compte du niveau organisationnel s'inscrit dans un processus évolutif de la littérature scientifique traitant de la problématique du turnover enseignant. En effet, si jusqu'au début des années 2000 la perspective d'analyse qui prédominait était centrée sur les individus et visait à déterminer dans quelle mesure les trajectoires professionnelles des enseignants étaient conditionnées par une série de facteurs individuels (sexe, ancienneté, discipline enseignée, etc.), depuis lors, elle a connu une inflexion significative au profit d'une approche considérant aussi les caractéristiques des organisations et de l'environnement de travail. Le spectre des variables mobilisées pour expliquer les phénomènes de mobilité professionnelle s'est dès lors enrichi mais, parallèlement, une forme de confusion a été introduite entre deux types de mobilité des enseignants pourtant distincts : le départ vers un autre établissement scolaire (migration professionnelle) et le départ vers une autre profession (attrition professionnelle). Ces deux situations ont les mêmes conséquences pour l'établissement qui perd un enseignant. Mais il est très probable que les motivations qui fondent ces comportements ne se recoupent que partiellement. Dans la présente note de synthèse, nous veillerons à distinguer au mieux les processus de migration et d'attrition professionnelles, mais notre commentaire sera parfois limité par les données disponibles dans les travaux consultés.

Signalons également que la quasi-totalité des études qui seront mentionnées dans cette note de synthèse portent sur un seul système éducatif ou sur un seul pays. Dès lors, par définition, elles sont peu propices à une élucidation des variations institutionnelles relatives aux modes de gestion des carrières, aux modalités de recrutement et/ou d'affectation des enseignants et aux conditions de rémunération, qui constituent des paramètres variant généralement peu au sein d'un même système éducatif. Elles ont donc pour tendance naturelle un penchant à négliger ces facteurs dont on pressent le rôle crucial dans la compréhension des conditions de mobilité des enseignants. Dans la présente note de synthèse, pour chacune des recherches mentionnées, nous spécifierons le système éducatif dans lequel elle a été réalisée. Toutefois, faute d'informations, nous ne serons pas toujours en mesure de documenter avec précision les conditions institutionnelles de gestion des carrières présentes dans ces derniers.

La question de la mobilité des travailleurs et du départ volontaire de leur lieu de travail n'est par ailleurs pas une question spécifique au champ scolaire. Elle a en effet fait l'objet de très nombreuses études dans d'autres secteurs professionnels et les cadres d'analyse mobilisés dans ces travaux viennent parfois irriguer les recherches menées sur les enseignants. Par conséquent, afin de rendre compte du développement de cette littérature, nous avons consacré la première section de cette note à une synthèse des études menées hors des contextes scolaires. L'essentiel de ce texte sera ensuite dédié à celles portant sur la mobilité professionnelle des enseignants, en distinguant fondamentalement les analyses centrées sur les caractéristiques de ceux-ci et celles qui ont mis en évidence l'influence des établissements scolaires et de leurs caractéristiques sur les taux de turnover.

Cette synthèse a été rédigée à partir d'articles scientifiques, d'ouvrages et de rapports de recherche européens et anglo-saxons traitant, de manière directe ou indirecte, de la problématique du turnover. Plus précisément, la revue de la littérature a été constituée sur la base d'un ensemble de mots-clés rédigés en français ou en anglais et recouvrant les principales thématiques abordées dans la note 2 . Ces mots-clés ont été encodés dans cinq moteurs de recherche ou bases de données : Google scholar, JSTOR, Science direct, Persée et Cairn. Les articles 
identifiés par cette voie ont souvent représenté, à travers leurs propres références bibliographiques, le point de départ de lectures complémentaires.

\section{RÉTENTION ET TURNOVER : UN OBJET DE RECHERCHE TRANSVERSAL}

Bien davantage que dans le champ scolaire, le départ volontaire des travailleurs est identifié de longue date comme un problème crucial dans la littérature relative à la gestion des ressources humaines (Holtom, Mitchell, Lee et al., 2008). Au regard du coût des processus de recrutement, du temps d'apprentissage des nouveaux travailleurs et de l'affaiblissement des réseaux interpersonnels au sein de l'organisation, la démission d'un travailleur est globalement perçue comme une pénalité pour l'employeur dont il s'agit de se préserver.

Très tôt, à partir des travaux précurseurs de March et Simon (1958), la satisfaction professionnelle (job satisfaction) a été identifiée comme une variable-clé associée à l'intention de quitter son emploi (Anderson \& Milkovich, 1980). Les modèles théoriques se sont ensuite progressivement complexifiés et ont également pris en considération des caractéristiques des individus (l'âge, l'ancienneté et le sexe en particulier), leur formation, leurs conditions de rémunération, les alternatives professionnelles perçues et des indicateurs relatifs à l'engagement organisationnel (organizational commitment), avant d'accorder davantage d'attention au contexte de travail (Holtom, Mitchell, Lee et al., 2008), lequel est appréhendé d'une part à partir des caractéristiques des organisations, mais aussi à partir de dimensions interpersonnelles et de perception du contexte. Concernant les organisations, c'est en particulier leur culture qui est étudiée, leur climat, le degré de cohésion en leur sein ou encore leur prestige. Concernant le rapport aux autres sur le lieu de travail, c'est davantage la perception du leadership, le soutien perçu et les relations interpersonnelles qui ressortent des investigations empiriques. Bien que les différents modèles théoriques les combinent de manière assez diversifiée, ces variables apparaissent comme étant statistiquement liées à l'intention de quitter un emploi et, quand les données le permettent, à la décision effective de changer de lieu de travail.

Cette complexification des modèles de prédiction n'empêche toutefois pas la littérature de continuer à considérer la satisfaction professionnelle et l'engagement organisationnel comme des prédicteurs du turnover d'une organisation ou des intentions individuelles de turnover. La satisfaction et l'insatisfaction professionnelles sont des réactions émotionnelles complexes qui résultent de l'interaction qui se produit entre un employé et l'environnement professionnel au sein duquel il évolue. Elles sont liées à l'évaluation que le personnel effectue entre ce qu'il souhaite professionnellement et ce que son emploi semble lui apporter. Trois éléments déterminent ce processus évaluatif. II s'agit de la perception des aspects de l'emploi par l'individu, de ses valeurs et standards et enfin de ses jugements conscients ou inconscients liés à la relation qui s'opère entre sa perception de l'emploi et ses valeurs (Locke, 1969). En ce qui concerne l'engagement organisationnel, il fait l'objet de plusieurs définitions. Certains auteurs se sont essentiellement basés sur les manifestations explicites de l'engagement et ont donc assimilé le concept à un comportement. D'autres, plus nombreux, l'ont plutôt défini en termes d'attitudes et ont mis en évidence la façon dont un individu s'identifie aux objectifs et valeurs d'une organisation et développe un sentiment d'appartenance destiné à en faciliter l'atteinte. Le concept inclut une relation d'échange au travers de laquelle le membre du personnel s'attache à l'organisation en retour de ressources octroyées par cette dernière. Dans cette optique, il réfère au degré d'identification et d'implication témoigné envers l'organisation 
et peut être mesuré par trois facteurs. II s'agit de l'acceptation de ses valeurs et objectifs, de la volonté de s'y investir et enfin du souhait de développer un sentiment d'appartenance (Mowday, Steers \& Porter, 1979).

Dans cette voie, un ensemble de recherches a consisté à mieux décrire et expliquer la nature des liens entre les caractéristiques des postes de travail et des organisations, les attitudes des individus, et le turnover (ou l'intention de turnover), montrant ainsi que les formes de leadership (Venkataramani, Green \& Schleicher, 2010), les modes de socialisation organisationnelle des employés (Boswell, Shipp, Payne et al., 2009), les « designs » de travail (Humphrey, Nahrgang \& Morgeson, 2007), ou encore les pratiques de gestion des ressources humaines (Weller, Holtom, Matiaske et al., 2009) ont un effet sur le turnover, qui passe par un renforcement de la satisfaction professionnelle et/ou de l'engagement organisationnel. Par ailleurs, la littérature en psychologie des organisations a tenté de mieux saisir comment s'imbriquent les relations entre la satisfaction professionnelle et l'implication organisationnelle. Elle pose ainsi la question de savoir dans quelle mesure les attitudes vis-à-vis du travail conditionnent l'implication envers l'organisation, et/ou dans quelle mesure l'intensité de l'engagement organisationnel colore avec plus ou moins de force le rapport des individus à leur travail. Par rapport à cette interrogation, les résultats d'études longitudinales (prenant plusieurs points de mesure de la satisfaction professionnelle, de l'engagement organisationnel et des intentions de turnover) semblent faire apparaître deux éléments principaux. D'une part, tant la satisfaction professionnelle que l'engagement organisationnel expliquent une part spécifique de la variance du turnover, et, d'autre part, ils se conditionnent mutuellement (Farkas \& Tetrick, 1989), signifiant ainsi que les voies d'action pour diminuer le turnover peuvent s'envisager aux deux niveaux (du poste de travail et de l'organisation) et que l'« entrée » par l'une peut être renforcée par un effet sur l'autre.

\section{RÉTENTION ET TURNOVER : DES RECHERCHES DANS LE CHAMP SCOLAIRE}

\section{Historique relatif à la littérature traitant de la problématique du turnover}

La question de la mobilité professionnelle des enseignants a donné lieu, en particulier depuis les années soixante-dix (Charters, 1970 ; Greenberg \& McCall, 1974 ; Mark \& Anderson, 1978), à une abondante littérature en économie et en sciences de l'éducation, essentiellement aux États-Unis. Cette dernière s'est principalement attelée, durant deux à trois décennies, à quantifier l'ampleur des sorties du métier (qualifiées d'attrition) et à décrire les facteurs qui les influencent. Elle a longtemps adopté une perspective d'analyse centrée sur les individus et leurs interactions avec le ou les marché(s) du travail, en cherchant à déterminer dans quelle mesure les trajectoires de maintien dans le métier sont conditionnées, d'une part par une série de facteurs individuels comme le sexe, le parcours de formation antérieur à l'entrée dans le métier, la discipline enseignée ou encore le niveau d'efficacité des enseignants une fois en classe, et d'autre part par des paramètres caractérisant le marché du travail enseignant et les marchés du travail vers lesquels se destinent potentiellement les individus quittant le métier d'enseignant, et en particulier les incitants monétaires (à se maintenir dans le métier ou à le quitter). La perspective économique dominante alors adopte un modèle d'analyse qui envisage l'individu comme un acteur rationnel posant des choix individuels et répondant à des incitants, soit à entrer et rester dans le métier d'enseignant, soit à occuper son temps d'une autre manière (Murnane, Singer \& Willett, 1987). 
L'émergence aux États-Unis de cette littérature consacrée à l'attrition des enseignants s'inscrit dans la poursuite d'une interrogation de longue date sur la considération des enseignants comme corps professionnel. En effet, depuis le XIXe siècle jusqu'aux années soixante, l'objet majeur du questionnement portait sur la qualité des recrues destinées à occuper les postes d'enseignant, en pointant notamment comme indicateurs de la faible qualité du recrutement le niveau limité de formation du personnel et l'important contingent féminin. Les années d'après-guerre ont toutefois largement changé la donne, en augmentant notamment de manière significative le niveau de qualification des futurs enseignants et en étant le théâtre d'une plus grande structuration des associations professionnelles en charge de représenter et défendre les intérêts des enseignants. Ces tendances de fond, conjuguées à des conjonctures de pénurie dans certains États américains, ont fait pencher la focale de la qualité des entrants vers leur maintien dans le métier (même si le débat relatif à la quantité et à la qualité des entrants dans le métier d'enseignant a continué à avoir une place et est toujours d'actualité aujourd'hui).

Cette centration de la littérature sur une perspective d'analyse individuelle d'inspiration économiciste a toutefois connu une inflexion significative au début des années 2000 (Ingersoll, 2001a) à la faveur, notamment, de nouveaux modes de régulation du curriculum et des carrières, qui accordaient une place plus importante aux établissements et à la gestion décentralisée. On notera également qu'avec cette inflexion de l'angle d'analyse, les recherches proprement scolaires sur la mobilité professionnelle ont suivi le même sentier de développement que les recherches de psychosociologie des organisations portant sur cette problématique, d'abord centrées sur les caractéristiques des individus, avant d'intégrer plus nettement les facettes occupationnelles et organisationnelles et leurs interactions avec les dimensions individuelles. Cette littérature se différencie de la perspective individuelle sur deux points principaux. Le premier concerne l'unité d'analyse : l'établissement scolaire, plus que les enseignants considérés individuellement, devient l'unité de référence principale à travers laquelle sont pensés et mesurés les problèmes de mobilité professionnelle. La conséquence en est que les phénomènes de mobilité interne au marché du travail enseignant (phénomènes de migration), tout autant que les sorties de ce marché du travail (phénomènes d'attrition), sont considérés avec une égale importance, puisque les deux types de mobilité professionnelle posent des problèmes similaires aux organisations scolaires en matière de recrutement, de stabilité des équipes éducatives ou encore de projets pédagogiques. Le second a trait aux variables mobilisées pour comprendre et expliquer les phénomènes de mobilité professionnelle : en plus des facteurs individuels et des caractéristiques des marchés du travail, au cœur des travaux précédents, sont pris en considération les caractéristiques des organisations et des environnements de travail. Des variables décrivant les modalités d'organisation du travail et la qualité des relations sociales au sein des établissements, comme le leadership de la direction ou le leadership partagé au sein de l'établissement, les modalités de prise de décision (plus ou moins concentrée, plus ou moins partagée) ou encore la collégialité au sein de l'équipe éducative, sont prises en compte pour expliquer les mouvements de départ des établissements, à côté de variables caractérisant davantage le contexte de l'établissement, comme la composition sociale des écoles et leur taille. L'opposition, ou plutôt la mise en contraste, de ces deux types d'explications organise d'ailleurs fortement ce nouveau pan de littérature, qui balance entre des interprétations du turnover en fonction des variables organisationnelles - considérant par là qu'à niveau égal de composition sociale des écoles, celles-ci peuvent faire la différence, y compris dans des écoles défavorisées socialement -, et des interprétations qui se concentrent prioritairement sur l'effet du public accueilli au sein des écoles. Des modèles multiniveaux sont utilisés pour estimer la part relative de l'explica- 
tion des sorties d'établissement liées respectivement aux facteurs individuels, aux caractéristiques des écoles (taille, niveau d'enseignement, composition sociale) et aux dynamiques organisationnelles. Cette littérature enrichit donc le spectre des variables mobilisées pour expliquer les phénomènes de mobilité professionnelle (même si certains travaux portant exclusivement sur l'attrition avaient déjà introduit de manière marginale comme variables explicatives des paramètres organisationnels). Elle tend cependant à introduire une forme de confusion entre les phénomènes d'attrition et de migration, regroupés derrière le concept et le terme de « turnover ", qu'elle ne dissocie plus, ni dans la mesure, ni dans la prédiction.

Pour compléter ce premier panorama des recherches relatives à la mobilité professionnelle des enseignants, on notera enfin que, parallèlement au développement de ces deux littératures traitant directement de l'attrition et de la migration des enseignants, ou des deux confusément, s'est développée une (ou des) littérature(s) se penchant sur le rapport subjectif des enseignants à leur métier et au contexte organisationnel dans lequel ils l'exercent. La question du rapport subjectif des enseignants à leur métier a été développée à travers les concepts de satisfaction (job satisfaction) et d'engagement (job involvment) professionnels, tandis que celle du rapport subjectif au contexte organisationnel d'exercice du métier l'a été via les concepts d'engagement (organizational commitment) ou d'identification (organizational identification) organisationnels. Bien que cet ensemble de travaux parfois hétérogènes se soit assigné des objectifs autres et plus larges que le seul objectif de prédire les types de mobilité professionnelle des enseignants, certaines études ont directement mis en relation ces concepts avec l'attrition des enseignants (Cha \& Cohen-Vogel, 2011) ou encore le turnover de ces derniers (Conley \& You, 2009). La connexion entre les appréhensions subjectives du métier ou du contexte de travail et les phénomènes de mobilité professionnelle apparaît donc bien plus lâche dans les sciences de l'éducation que dans les approches psychosociologiques, qui avaient fait de ces concepts des explications premières de la mobilité professionnelle et ont continué d'y porter une attention importante, en considérant cette fois la satisfaction professionnelle ou l'engagement organisationnel comme des processus médiateurs de l'influence des paramètres de contexte organisationnel ou de travail sur la mobilité des travailleurs.

Dans la suite de cette revue de la littérature, nous chercherons à identifier les facteurs individuels - tant objectifs que subjectifs - et organisationnels qui influencent le turnover enseignant.

\section{Les facteurs individuels}

\section{Les facteurs objectifs}

\section{L'ancienneté des enseignants}

La littérature américaine met en exergue que la distribution du turnover présente la forme d'une parabole en $U$ sur l'ensemble du personnel enseignant étudié à travers le prisme de son ancienneté (Ingersoll, 2001a). Ainsi, diverses recherches menées au cours des trois dernières décennies dans plusieurs États américains stipulent que, quel que soit le niveau d'enseignement au sein duquel les enseignants professent, les phénomènes de migration et d'attrition sont effectivement plus marqués parmi ceux qui débutent leur carrière professionnelle et parmi ceux qui approchent de la retraite (Adams, 1996 ; Kukla-Acevedo, 2009 ; Luekens, Lyter \& Fox, 2004 ; Theobald, 1990). 
En ce qui concerne les taux d'abandons précoces, les données collectées au sein des cinquante États américains par le National Center for Education Statistics dans le cadre de quatre cycles relatifs à la School And Staffing Survey (SASS) s'étalant de 1987-1988 à 1999-2000 mentionnent que $11 \%$ des enseignants ${ }^{3}$ quittent la profession au cours de la première année et $39 \%$ dans les cinq ans (Ingersoll, 2002). À ce titre, selon Kirby, Berends et Naftel (1999), les taux d'attrition observés au niveau national ont légèrement diminué entre le milieu des années quatre-vingt et la fin des années quatre-vingt-dix. L'exposition élevée des enseignants débutants au risque de sortie du métier peut être partiellement expliquée par les conditions administratives de gestion des carrières qui font souvent peser sur les nouveaux entrants dans la profession l'instabilité d'emploi et des conditions de travail plus difficiles. Dans de nombreux pays, ce n'est en effet qu'après quelques années de travail que les enseignants ont la possibilité de se stabiliser dans un établissement plus ou moins choisi. Mais une telle explication n'épuise pas la problématique et il est probable que ce départ accru des enseignants novices repose également sur des difficultés d'entrée dans le métier et des tensions entre l'image initiale de ce métier et les conditions pragmatiques de son exercice (Devos, 2012).

Les données empiriques disponibles pour la Belgique révèlent une situation semblable. En effet, $40 \%$ des enseignants ayant exercé au sein de la Fédération Wallonie-Bruxelles (enseignement secondaire) entre 1973 et 1997 et 33,5\% de leurs collègues ayant intégré un établissement néerlandophone ${ }^{4}$ entre 1999 et 2008 ont quitté la profession avant d'avoir atteint leur sixième année d'ancienneté (Vandenberghe, 2000 ; Vlaams Ministerie van Onderwijs en Vorming, 2009). Plus récemment, une recherche menée en Belgique francophone sur des données s'étalant de septembre 2005 à août 2009 (Delvaux, Desmarez, Dupriez et al., à paraître) précise que les taux de sortie en début de carrière ne sont pas identiques au sein des différents niveaux d'enseignement. Ainsi, si $45 \%$ des enseignants professant dans l'enseignement secondaire (tous types confondus) quittent la profession au cours des cinq premières années d'exercice, ces départs précoces concernent moins de $30 \%$ des individus engagés au sein de l'enseignement fondamental (préscolaire et primaire). De plus, outre le niveau d'enseignement, la détention d'un diplôme pédagogique limite de manière significative les risques de départ en début de carrière. En effet, le taux cumulé d'abandon au terme de la cinquième année d'exercice est trois fois plus élevé (60\% contre $20 \%$ ) parmi les enseignants n'ayant pas reçu de formation à caractère pédagogique et n'étant, dès lors, pas titulaires du titre requis. Or, dans plusieurs pays européens tels que la Belgique (toutes communautés linguistiques confondues), l'Allemagne, le Luxembourg, les Pays-Bas et le Royaume-Uni (European Commission, 2012), un pourcentage non négligeable ${ }^{5}$ d'établissements se voit confronté à une pénurie manifeste dans certaines disciplines et doit confier ses classes à des enseignants non qualifiés pour l'enseignement de la matière attribuée.

Ces sorties fréquentes du métier caractérisant les jeunes enseignants sont le plus souvent imputées à la trajectoire professionnelle précaire qui caractérise le personnel débutant ainsi qu'aux affectations fréquentes dans des établissements proposant des conditions de travail plus difficiles que ce dernier subit (Greenberg \& McCall, 1974 ; Mukamurera, 1999 ; Rayou \& van Zanten, 2004 ; Loeb, DarlingHammond \& Luczak, 2005). En effet, tant en Amérique du Nord qu'en Europe (Léger, 1981 ; Hart \& Murphy, 1990 ; Mukamurera, 1999 ; Vandenberghe, 2000), vu le poids de l'ancienneté comme source de priorité pour les affectations des enseignants, rares sont les jeunes enseignants qui décident librement de leur lieu de travail. Ils se voient souvent contraints à cumuler les contrats à durée déterminée ou à temps partiel, à répartir leur temps de travail sur plusieurs implantations scolaires, 
à enseigner des matières éloignées de leur formation initiale et à professer loin de leur ville voire de leur région d'origine. De plus, ils sont surreprésentés au sein des établissements accueillant un public en tension avec les normes scolaires et souvent fuis par les membres du personnel chevronnés. La conjonction de ces éléments peut conduire les plus inexpérimentés à se décourager lors de la phase de première insertion professionnelle qui est pourtant prépondérante pour la construction de l'identité professionnelle (Maroy \& Cattonar, 2002). Dans ce contexte, les membres du personnel qui se maintiennent dans le métier intègrent souvent la possibilité d'une mobilité horizontale (se manifestant essentiellement par un changement d'établissement) dans leurs projets de carrière, en envisageant leur trajectoire professionnelle en fonction de leurs attentes et non selon les parcours explicitement dessinés par l'institution et le cadre réglementaire en vigueur (Rayou \& van Zanten, 2004).

Ce phénomène de mobilité horizontale n'est pas propre aux premières années de carrière et concerne également les enseignants qui ont dépassé le seuil critique de l'entrée dans le métier. La profession enseignante se distingue en effet de nombreuses autres professions par une importance particulière accordée aux carrières horizontales étant donné la faible diversité de postes aux niveaux supérieurs et, dès lors, la probabilité peu élevée de pouvoir ambitionner une mobilité verticale (Rayou \& van Zanten, 2004 ; Léger, 1983). Globalement, après quelques années d'ancienneté, la grande majorité des enseignants se maintient dans la profession sur le long terme (Adams, 1996). Certains renoncent toutefois à leurs attributions pour des raisons professionnelles ou personnelles. Ainsi, au travers d'une recherche menée en Norvège sur des enseignants exerçant au sein des niveaux fondamental et secondaire inférieur au cours des années scolaires s'étalant de 1992-1993 à 1999-2000, Falch et Strom (2005) ont mis en exergue que les femmes en âge d'avoir de jeunes enfants sont davantage enclines à s'écarter de la profession. Ce comportement est facilité par les politiques en vigueur en Norvège et plus largement dans les pays scandinaves qui facilitent les interruptions de carrières destinées à l'éducation des enfants. Toutefois, bien que le contexte y soit moins favorable, Evetts (1988) et Murnane et Olsen (1989) ont également mis en évidence cette tendance à délaisser la fonction enseignante pour se concentrer sur la famille, respectivement au Royaume-Uni et aux États-Unis. Toutefois, il est à noter que lorsque le départ de la profession n'est pas consécutif à une insatisfaction ou à des opportunités de carrière externes à l'enseignement, nombreux sont les individus qui la réintègrent ultérieurement (Boe, Bobbitt, Cook et al., 1998).

Enfin, comme précisé ci-dessus, les taux de sortie du métier s'élèvent à nouveau lorsque l'on considère les membres du personnel les plus expérimentés qui délaissent leur charge d'enseignement pour prendre une retraite anticipée ou pour partir à la retraite (Boe, Bobbitt, Cook et al., 1998). Aux États-Unis, au cours de l'année scolaire 1994-1995, les départs à la retraite représentaient $12 \%$ du turnover global (migrations et attritions) et un départ définitif de l'enseignement sur quatre (Ingersoll, 2001b).

La formation initiale, la discipline enseignée et les facteurs sociodémographiques relatifs aux enseignants

La littérature considère que l'ancienneté n'est pas l'unique facteur individuel objectif qui influence les taux de turnover des enseignants et que d'autres caractéristiques, telles que la formation, la discipline enseignée et les facteurs sociodémographiques ont également un impact.

En ce qui concerne la formation, de part et d'autre de l'Atlantique, de nombreux auteurs ont souligné son influence sur la stabilité professionnelle des enseignants. Ainsi, Boe, Bobbitt, Cook et aliib (1997) ont mené une recherche portant sur le turnover des enseignants professant au sein des enseignements général et spécialisé aux 
États-Unis et montrent que ceux qui possèdent les titres requis pour l'enseignement de la discipline qui leur est attribuée ont davantage tendance à se maintenir dans l'établissement au sein duquel ils sont insérés et, plus largement, dans la profession. Dolton et van der Klaauw ${ }^{7}$ (1999) montrent également qu'à niveau de formation initiale identique, l'orientation pédagogique ou non pédagogique du diplôme influe sur la stabilité professionnelle. Leurs données, portant sur les sept premières années de carrière de diplômés d'universités anglaises, soulignent que les individus ayant suivi une formation à caractère pédagogique ont moins tendance à quitter le secteur de l'enseignement que leurs collègues possédant un diplôme ne provenant pas d'une filière pédagogique. Ces derniers détiennent en effet un titre ne prédisposant pas nécessairement à l'enseignement et dont la valeur dans d'autres secteurs professionnels peut être plus importante. Ils sont également moins préparés à faire face aux exigences du métier. Dans le même ordre d'idées, Falch et Strom ${ }^{8}$ (2005) mettent en évidence, sur la base de données norvégiennes, que le niveau d'études a également un impact sur le turnover. Le personnel qui a obtenu un titre au terme de longues années d'études présente une propension plus élevée à la mobilité professionnelle. Les auteurs expliquent ce constat par le fait que les individus qui ont poursuivi des études au-delà des années de formation destinées à l'enseignement ont acquis des compétences supplémentaires les rendant davantage attractifs sur le marché de l'emploi et leur permettant de négocier plus aisément leur rémunération au sein d'autres secteurs professionnels. Les données recueillies par Schlechty et Vance (1981) en Caroline du Nord (États-Unis) apportent des éléments complémentaires et soutiennent également que le personnel le plus diplômé est davantage enclin à quitter précocement la profession enseignante. En Belgique francophone, l'influence majeure du diplôme a également été mise en exergue sur la base de données récentes portant sur des individus ayant débuté leur carrière enseignante entre septembre 2005 et août 2009 (Delvaux, Desmarez, Dupriez et al., à paraître). En effet, comme mentionné ci-dessus, la possession du titre requis pour l'enseignement de la matière attribuée et, plus largement, d'un diplôme pédagogique, constitue un facteur central de stabilité professionnelle. Par ailleurs, parmi les titulaires d'un diplôme pédagogique, cette étude belge montre que les individus ayant obtenu leur diplôme au terme d'études universitaires de longue durée présentent une propension plus importante à quitter précocement le secteur de l'enseignement. Ce constat peut, au moins en partie, être imputé à la formation plus généraliste reçue par ces membres du personnel et aux opportunités d'emplois alternatives plus nombreuses qui s'offrent à eux.

Certaines fonctions sont également plus exposées à un turnover élevé. En effet, selon Boe, Bobbitt, Cook et alii (1997), les établissements d'enseignement spécialisé rencontrent des difficultés plus importantes à stabiliser leur personnel. Leurs résultats se basent sur des données administratives récoltées sur l'ensemble du territoire américain à la fin des années quatre-vingt et montrent que la rétention annuelle est de $88 \%$ dans les établissements dispensant un enseignement général et seulement de $80 \%$ au sein de ceux destinés à l'enseignement spécialisé ${ }^{9}$. Par ailleurs, sur la base de données provenant de l'État américain de l'Indiana, Grissmer et Kirby (1992) constatent que l'enseignement secondaire est davantage victime de taux élevés d'abandon et que les professeurs de sciences et, dans une moindre mesure, de mathématiques et de langue maternelle sont les plus instables. Plus précisément, après avoir contrôlé le genre et l'âge de l'individu à l'entrée, les cours de chimie et de physique subissent les taux de départs les plus importants, suivis, dans l'ordre, de ceux de biologie, de langue maternelle et de mathématiques. Ces mêmes résultats ressortent d'autres recherches nord-américaines (Levin, 1985 ; Rumberger, 1987). Plus récemment toutefois, aux États-Unis également, Ingersoll (2000) a effectué une recherche relative aux enseignants dispensant les cours de sciences et de mathématiques et a mis en exergue que, si les taux de migration 
et d'attrition sont légèrement plus élevés pour ces disciplines, au regard de ses données, les différences observées ne sont pas statistiquement significatives. Bien que ces résultats de recherches aient été obtenus au sein de systèmes éducatifs spécifiques ne permettant pas une généralisation aisée à d'autres contextes, ils soulignent que tous les niveaux d'enseignement et toutes les disciplines dispensées ne sont pas égaux face à la problématique du turnover.

Par ailleurs, les caractéristiques sociodémographiques, telles que le genre et l'origine ethnique des enseignants, constituent également un facteur ayant un lien avec le turnover. L'impact du genre conduit à des résultats contrastés au sein de la littérature. Ainsi, sur la base de données administratives provenant de la Schools and Staffing Survey 1990-1991 (SASS) et de la Teacher Followup Survey 1991-1992 (TFS) et portant sur l'ensemble des États-Unis, Ingersoll10 (2001b) a mis en exergue que, dans les établissements privés, les enseignantes présentent davantage de stabilité que leurs collègues de sexe masculin alors que le phénomène inverse s'observe au sein des écoles publiques. Strunk et Robinson ${ }^{11}$ (2006) se sont penchés sur des données récoltées dans le cadre des mêmes enquêtes quelques années plus tard (SASS 1999-2000 et TFS 2000-2001). Bien qu'ils ne distinguent pas le caractère public ou privé de l'établissement, ils ne font ressortir aucune influence significative du genre sur les taux d'attrition ou de migration des enseignants. Ces taux similaires de sorties selon le sexe ont également été mis en évidence par Rees (1991; cité par Newton, Rivero, Fuller et al., [s.d.]) dans le cadre d'une recherche portant sur des écoles publiques situées dans l'État américain de New York; cependant, l'auteur précise qu'une fois mères, les enseignantes tendent davantage à quitter temporairement ou définitivement la vie active pour se consacrer à leur famille. À l'inverse, en France, sur la base d'un échantillon représentatif des enseignants exerçant dans l'enseignement secondaire, Périer (2003) stipule quant à lui que l'intention de quitter la profession apparaît plus spécifique aux hommes qu'aux femmes.

En outre, en ce qui concerne l'origine ethnique 12 des enseignants, si l'analyse de son impact sur le turnover a reçu une attention particulière aux États-Unis, elle est nettement moins étudiée dans le cadre d'études européennes. Ainsi, Kirby, Berends et Naftel (1999) ont mis en évidence que sur le territoire américain, au cours de l'année scolaire 1993-1994, 13,5 \% de l'ensemble du personnel enseignant était issu de minorités ethniques. Plus particulièrement, $6,7 \%$ des enseignants étaient d'origine afro-américaine, 4,1\% étaient hispano-américains et 1,7 \% provenaient d'autres minorités ethniques. Ces mêmes auteurs ont réalisé une enquête sur la base de données longitudinales portant sur des enseignants texans ayant professé entre 1979 et 1996 et soulignent qu'alors que le personnel hispano-américain présentait des taux d'attrition inférieurs de $16 \%$ à ceux observés pour leurs collègues américains blancs, aucune différence statistiquement significative ne pouvait être observée concernant l'écart entre ces derniers et les individus afro-américains. Murnane et Olsen (1989) ont quant à eux étudié les enseignants du Michigan et mettent en exergue que les enseignants afro-américains ont tendance à se maintenir davantage dans la profession. Cette propension se manifeste également dans les résultats obtenus par Adams (1996) auprès d'individus professant au sein d'établissements du niveau fondamental au Texas montrant que le personnel issu de minorités ethniques est plus stable que son homologue américain blanc.

\section{Les dimensions subjectives}

\section{La satisfaction professionnelle}

Au-delà des dimensions objectives liées au turnover enseignant, la littérature dans le champ scolaire a également considéré le rôle joué par des facteurs subjectifs comme le rapport des enseignants à leur métier (à travers le concept de satisfaction 
professionnelle) ou à leur établissement (à travers le concept d'engagement organisationnel). Le plus souvent, ce pan de littérature n'a pas directement établi de lien entre les concepts de satisfaction professionnelle ou d'engagement organisationnel et le turnover. En effet, le parti pris par cette voie de recherche dans le champ scolaire est différent : plutôt que de mesurer le lien entre le turnover et les attitudes des enseignants, la majorité des recherches dans le champ scolaire prennent ce lien pour un acquis de la littérature (à l'instar de ce que nous avons présenté dans la première section de cette note) et tentent de comprendre les variations observées chez les enseignants dans leurs niveaux de satisfaction professionnelle et/ou d'engagement envers leur établissement.

Lawler et Porter (1967 ; cités par Taylor et Tashakkori, 1995) mettent à cet égard en évidence que la satisfaction professionnelle est associée à des ressources intrinsèques et extrinsèques. Les premières émanent de l'enseignant et sont liées à ses caractéristiques individuelles ainsi qu'aux sentiments d'accomplissement et de performance qu'il peut éprouver. Les secondes se caractérisent par un ensemble d'éléments liés à l'organisation et, plus largement, au système, tels que le salaire ou les promotions envisageables. Vu les modes de gestion des carrières dans l'enseignement et le peu d'incitants financiers en présence, le personnel a historiquement été contraint de délaisser les sources de ressources extrinsèques et de se tourner vers celles qui lui sont intrinsèques comme la satisfaction que lui procure l'exercice du métier (Lortie, 1975 ; cité par Taylor et Tashakkori, 1995). Cependant, suite à la massification scolaire, la tâche d'enseignement s'est complexifiée et cette source de satisfaction s'est affaiblie, surtout dans les établissements au sein desquels le public accueilli est en tension avec les valeurs scolaires (Taylor \& Tashakkori, 1995). Cette difficulté concourt, au minimum indirectement, au taux de turnover important dont sont victimes certains établissements d'enseignement et aux nombreux départs précoces que connaît la profession (Ingersoll, 2001b).

De nombreux auteurs ont par ailleurs discuté l'impact d'un ensemble de caractéristiques propres à l'individu sur sa satisfaction professionnelle. Des recherches ont par exemple affirmé l'existence de différences significatives en termes de satisfaction professionnelle selon le genre, en mettant en exergue que les attentes formulées à l'égard de la carrière ne sont pas identiques. Toutefois, les conclusions ne vont pas toutes dans la même direction. Si Huberman (1993) et Ma et MacMillan (1999) estiment que de manière générale les enseignantes professant respectivement dans l'enseignement secondaire en Suisse et dans l'enseignement fondamental au Canada sont plus satisfaites que leurs collègues de sexe masculin, d'autres chercheurs canadiens et américains (Liu \& Ramsey, 2008 ; Klassen \& Chiu, 2010) soutiennent que les femmes sont moins satisfaites professionnellement et ce, de par une perception souvent plus négative des conditions de travail et un stress plus marqué en réponse à la charge de travail qui leur est imputée. L'expérience professionnelle conditionne également la satisfaction professionnelle. Toutefois, pour ce facteur également, les chercheurs qui se sont penchés sur la problématique ne sont pas unanimes quant au niveau d'ancienneté associé à la satisfaction professionnelle la plus élevée. Si selon une étude menée en Suisse par Huberman (1993), l'individu en fin de carrière serait moins satisfait de son emploi que ses collègues moins expérimentés, Wisniewski (1990) déclare, sur la base d'une recherche menée auprès d'enseignants polonais, que l'individu plus chevronné est plus satisfait professionnellement. De même, Menon et Christou (2002) montrent, à travers une recherche menée à Chypre, que le personnel débutant manifeste un taux plus élevé d'insatisfaction suite à un décalage entre ses perceptions du travail et les attentes développées au cours de sa formation. 
La discipline enseignée peut aussi affecter la satisfaction professionnelle. En effet, bien que, comme développé précédemment, les enseignants dispensant des matières scientifiques soient davantage enclins à réorienter leur carrière, ces disciplines sont souvent mieux considérées socialement et des ressources plus importantes sont parfois allouées à leur enseignement. De plus, elles sont souvent pensées comme nécessitant davantage d'attention et de travail émanant des élèves. Cela engendre, semble-t-il, un sentiment d'efficacité plus élevé et, corollairement, davantage de satisfaction émanant du membre du personnel (Bishay, 1996).

La satisfaction professionnelle du personnel enseignant semble donc être conditionnée par un ensemble de caractéristiques liées au sexe, à l'ancienneté, à la discipline enseignée et aux conditions de travail. Toutefois, la littérature fait apparaître des résultats de recherche assez diversifiés en fonction des études et des systèmes éducatifs considérés.

\section{L'engagement organisationnel}

Un autre indicateur de nature subjective régulièrement considéré par la littérature est l'engagement organisationnel des enseignants envers leur établissement. Au sein du secteur de l'enseignement, l'engagement organisationnel a été étudié de deux manières. D'une part, positivement, par l'intermédiaire de l'identification et de l'attachement professionnels manifestés par le personnel. Ceux-ci sont fortement corrélés à une implication et à un investissement élevés dans les tâches à effectuer et à un engagement important envers l'organisation (Tyree, 1996). D'autre part, plus négativement, au travers du manque d'engagement. Cette attitude de retrait est généralement interprétée comme l'expression d'un malaise professionnel qui entrave l'adhésion aux valeurs et objectifs propres à l'établissement scolaire et, plus largement, à la profession (Blau \& Boal, 1987 ; Guillemette, 2006).

Par rapport à cette thématique, la littérature américaine a en particulier montré que le degré d'engagement d'un enseignant n'est pas stable tout au long de la carrière. De même, ce que l'enseignant attend de son lieu de travail évolue avec les étapes de sa carrière. De manière générale, l'enseignant débutant peine à outrepasser ses intérêts personnels et cherche des solutions immédiates aux difficultés qu'il rencontre. II se voit souvent confronté à des sentiments d'incertitude, de confusion et d'insécurité qui l'incitent à se focaliser sur les éléments qui entravent le déroulement des tâches quotidiennes (Chapman, 1983). Dès lors, son engagement envers l'organisation est fragile et dépend fortement des conditions de travail propres à l'établissement d'enseignement. L'individu qui bénéficie de quelques années d'ancienneté a, quant à lui, appris à faire face aux difficultés qui caractérisent la profession. Bien que les caractéristiques du système dans lequel il est inséré demeurent influentes, son engagement en est moins tributaire. En ce qui concerne le personnel qui a acquis plus d'une dizaine d'années d'expérience, il est également davantage influencé par les facteurs organisationnels liés aux tâches d'apprentissage (Rosenholtz \& Simpson, 1990). Son engagement est guidé par une volonté de développement professionnel et une crainte de la monotonie et du désintéressement des élèves lié à l'installation d'une routine dans la manière d'aborder la discipline enseignée (Blase, 1986).

Par ailleurs, l'influence du genre a également été étudiée, mais demeure sujette à discussion. Coladarci (1992) atteste que les enseignantes manifestent un engagement plus marqué que leurs homologues de sexe masculin. Par contre, Evans et Tribble (1986) n'ont mis en évidence aucune incidence significative du genre sur l'engagement. 

des enseignants

\section{La composition sociale, ethnique et académique des établissements}

Tout d'abord, les caractéristiques sociodémographiques propres à la population scolaire d'un établissement donné influencent les conditions de travail et prédisent une part importante des variations relatives au turnover. De nombreuses études montrent en effet que les établissements d'enseignement accueillant une large proportion d'élèves socio-économiquement défavorisés, issus de minorités ethniques et/ou présentant en moyenne des difficultés importantes d'apprentissage sont davantage sujets à des taux de turnover élevés de la part de leur personnel. À partir d'un échantillon d'écoles publiques primaires et secondaires texanes, Hanushek, Kain et Rivkin (2004) ont mis en exergue que, quelle que soit l'ancienneté, les taux de migration et d'attrition des enseignants dépendent fortement des performances moyennes des élèves et du pourcentage d'élèves d'origine afro-américaine ou hispano-américaine. Plus précisément, d'une part un taux élevé de réussite influence significativement et positivement la stabilité du membre du personnel et, d'autre part le personnel américain blanc est davantage enclin à quitter l'établissement voire la profession lorsque l'établissement accueille une proportion importante d'élèves issus de minorités ethniques. Des tendances similaires ont également été observées dans plusieurs systèmes éducatifs européens (van Zanten \& Grospiron, 2001 ; Falch \& Strom, 2005) tel le système français. Ainsi, sur la base d'une recherche portant sur les enseignants du secondaire, Léger (1983) a mis en exergue que la vitesse moyenne de rotation variait fortement selon le type social de l'établissement, un lycée très populaire ${ }^{13}$ se voyant contraint à renouveler les deux tiers de son personnel enseignant tous les quatre ans alors qu'un lycée très bourgeois ${ }^{14}$ ne doit remplacer qu'un peu plus du tiers de son effectif au cours de la même période.

Toutefois, la tendance inverse s'observe parmi les enseignants d'origine étrangère qui se maintiennent davantage au sein d'écoles scolarisant de nombreux élèves issus de l'immigration. C'est ce que suggèrent deux chercheurs américains, Greenberg et McCall (1974) en soulignant que le personnel afro-américain ou hispano-américain marque une préférence pour professer au sein d'établissements scolaires accueillant une proportion importante d'élèves issus de l'immigration, souvent socio-économiquement défavorisés, et que leur propension à demander un changement d'affectation pour un établissement scolaire plus aisé est moindre. Shen (1997), Scafidi, Sjoquist et Stinebrickner (2007), de même que Jackson (2009), abondent aussi dans ce sens sur la base de données empiriques issues respectivement de l'ensemble du territoire des États-Unis et des États de Géorgie et de Caroline du Nord.

Par ailleurs, d'autres recherches ont également mis en évidence que les compétences et les qualifications des enseignants sont aussi liées aux attributs ethniques, économiques et scolaires caractérisant les élèves d'un établissement. À ce titre, sur la base d'une recherche menée dans l'État de New York aux États-Unis, Lankford, Loeb et Wyckoff (2002) ont montré que les élèves issus de l'immigration, socioéconomiquement défavorisés ou présentant des difficultés scolaires, sont souvent confiés à des individus moins qualifiés voire non qualifiés pour l'enseignement de la matière qui leur est attribuée. Ils expliquent ce constat par le fait que, généralement, les enseignants - et surtout ceux qui bénéficient de plus de qualifications - ont tendance à quitter les établissements scolaires défavorisés. Or, ce comportement n'est pas sans conséquence et engendre des disparités importantes entre écoles en termes de qualité de l'enseignement. Ces mêmes conclusions ont également été exposées en Europe, et plus spécifiquement en Norvège. Bonesronning, Falch et Strom (2005) ont exploité une base de données administrative fournissant des informations pour les enseignants professant dans l'enseignement fondamental et 
secondaire inférieur. Leurs résultats montrent que le personnel pleinement qualifié marque une préférence pour travailler avec des enfants ou des adolescents non issus de minorités ethniques et ne présentant pas de besoins spécifiques.

\section{Les caractéristiques organisationnelles}

Les établissements scolaires ne sont pas seulement victimes des tendances démographiques et des caractéristiques de leur public. Les caractéristiques et les pratiques organisationnelles développées en leur sein sont également associées à la mobilité professionnelle de leur personnel. La littérature met ainsi en exergue qu'un ensemble de facteurs différencient les établissements scolaires et influencent, de manière directe ou indirecte, les choix de carrière posés par les enseignants. À ce titre, la littérature internationale tend à montrer que les établissements caractérisés par certaines formes de leadership et prônant le développement d'un travail collégial entre l'ensemble des membres du personnel attirent davantage les enseignants (Johnson, Berg \& Donaldson, 2005).

Plusieurs recherches adossées au paradigme du leadership transformationnel, promouvant l'instauration et le maintien d'un travail collectif, le développement professionnel du personnel et la résolution des problèmes rencontrés (Leithwood \& Poplin, 1992), attirent ainsi l'attention sur les capacités de la direction à soutenir et à épauler les membres du personnel (Ladd, 2011 ; Dumay \& Galand, 2012). De même, elles mettent l'accent sur l'importance du développement d'un processus de «teacher empowerment ». Ce dernier est un processus selon lequel «les enseignants développent des compétences destinées à influencer leur évolution et à résoudre les problèmes qu'ils rencontrent " en bénéficiant d'une autonomie dans le travail tant au niveau de la classe que de l'école (Short, Greer \& Melvin, 1994, p. 38 ; cités par Bogler \& Somech, 2004).

Cette valorisation de l'autonomie des enseignants dans leur travail et de leur participation aux prises de décision relatives à l'établissement - telles que la participation à l'organisation des horaires de cours ou à la sélection du matériel - tend à diminuer le taux de turnover ${ }^{15}$ (Johnson, 2006 ; cité par Boyd, Grossman, Ing et al., 2011). C'est également, sur la base de données récoltées sur l'ensemble du territoire américain, ce que montre Ingersoll16 (2001a) : après avoir contrôlé les caractéristiques à la fois de l'enseignant et de l'établissement, une relation négative est observée entre d'une part l'autonomie et la participation aux décisions et d'autre part le taux de turnover du personnel.

La direction joue également un rôle dans l'épanouissement professionnel des enseignants en les soutenant dans l'exercice de leurs fonctions. En effet, une assistance et une communication destinée à traiter les problèmes pédagogiques, de discipline, de curriculum ou d'ajustement vis-à-vis du milieu scolaire diminuent les taux de migration et d'attrition des enseignants et plus particulièrement de ceux qui débutent dans la profession (Ingersoll, 2001a). De manière plus générale, la mise en place d'un travail collectif entre le personnel enseignant, auxiliaire et administratif constitue aussi un facteur contribuant au bon fonctionnement de l'établissement (Johnson, Berg \& Donaldson, 2005). Allensworth, Ponisciak et Mazzeo17 (2009) ont à ce propos analysé la mobilité enseignante au sein des écoles publiques de Chicago (États-Unis) et mettent en évidence qu'alors que les conflits internes ont un impact négatif sur la stabilité du personnel, la manifestation d'une solidarité provoque l'effet inverse en influençant positivement l'ambiance de travail et en incitant les différents corps professionnels à collaborer. À cet égard, mentionnons toutefois les résultats d'une récente recherche menée en Belgique francophone (Devos, Dupriez \& Paquay, 2012) qui nuance quelque peu l'analyse des effets de la collaboration entre enseignants. Cette recherche montre en effet que l'intensité des échanges entre collègues 
n'a pas systématiquement une influence positive sur les enseignants (la variable de référence est ici le sentiment d'efficacité personnelle des enseignants). Par exemple, les enseignants débutants rencontrant de nombreuses difficultés dans leur travail vont se sentir moins efficaces dans un environnement caractérisé par de nombreuses interactions avec les collègues. Ce dernier résultat s'explique probablement par le fait que les échanges entre enseignants contribuent également à rendre plus visibles les faiblesses de chacun et que celles-ci ne pourront être dépassées que si ce même environnement propose réellement un soutien pour surmonter ces difficultés.

\section{Les caractéristiques du travail}

L'enseignant passant la majeure partie du temps dédié à sa vie professionnelle au sein de la classe, les relations entretenues avec les élèves occupent une place primordiale dans son épanouissement professionnel. En effet, la présence d'un climat favorable entre l'enseignant et les élèves dont il a la charge constitue également un élément influençant les taux de migration et d'attrition du personnel (Ingersoll, 2001a). Or, suite à la massification de l'enseignement, le public scolaire est devenu plus hétérogène et plus « difficile ". Ainsi, comme le soulignent Maroy et Cattonar (2002, p. 45), " les conditions sociales et culturelles qui favorisaient auparavant la participation et l'écoute des élèves, et encourageaient un rapport positif au savoir ne sont plus données a priori par le contexte familial et social des élèves ". Bien que son intensité soit variable selon les établissements d'enseignement, une culture juvénile se développe en tension avec les normes et les pratiques véhiculées par l'institution scolaire. Elle établit un sentiment de distance tant sociale que culturelle avec les enseignants dont la culture de référence reste attachée au modèle antérieur. En France, certains auteurs considèrent que cette distance s'est progressivement exacerbée car le recrutement social du corps des instituteurs et des professeurs du secondaire s'est élevé au cours de ces dernières décennies (Barrère \& Martuccelli, 1996). Ce comportement problématique de certains jeunes envers leur scolarité conditionne la nature du travail à fournir par l'enseignant et influe fortement sur sa satisfaction, son engagement, sa motivation et, corollairement, son intention de se maintenir au sein de l'établissement voire de la profession. À cet égard, sur la base de données récoltées sur l'ensemble du territoire américain, Ingersoll18 (2001a) explique que les problèmes de discipline et de respect des normes scolaires ont une influence sur les taux de turnover.

Ladd $^{19}$ (2011) a pour sa part travaillé sur des enseignants américains exerçant en Caroline du Nord et conclut de manière similaire. En effet, elle atteste que les caractéristiques comportementales et scolaires propres aux élèves influencent la stabilité professionnelle par l'intermédiaire de deux mécanismes principaux. D'une part, les élèves manifestant un manque de respect, une absence de discipline et/ou un désintéressement pour la matière enseignée peuvent mettre le membre du personnel dans une situation inconfortable l'empêchant de s'épanouir professionnellement sur le long terme. D'autre part, la prise en charge d'enfants ou de jeunes affichant des lacunes importantes en termes d'apprentissage peut engendrer un sentiment d'inefficacité conduisant à un découragement. Toutefois, Maroy et Cattonar (2002), à propos de la Belgique, modèrent ce constat en stipulant que le personnel qui professe dans un établissement au sein duquel le retard moyen par élève est plus important s'accoutume davantage à la complexité de sa fonction et témoigne d'un contentement plus élevé face à des difficultés similaires. Dans le même ordre d'idées, en France, une partie des jeunes enseignants considère que les établissements qualifiés de "difficiles " peuvent constituer des laboratoires permettant d'apprendre à maîtriser toutes les dimensions de la profession et ne cherche pas à les fuir de manière précipitée (Pochard, 2008). Ces conclusions contrastées doivent probablement à nouveau être analysées en gardant en mémoire 
les environnements institutionnels spécifiques de ces différentes recherches. Le contexte américain (voir Meuret, 2007) se caractérise en effet depuis un peu plus d'une décennie par des normes et des dispositifs de redevabilité particulièrement exigeants envers les établissements scolaires, allant même jusqu'à la menace de fermeture de l'établissement qui aurait sous-performé durant cinq années consécutives. On peut imaginer l'influence de tels dispositifs sur les enseignants travaillant avec des élèves dont les résultats sont largement inférieurs aux moyennes régionales. Les analyses de Maroy et Cattonar (2002) ainsi que celles de Pochard (2008) s'inscrivent par contre dans les environnements belge et français du début des années 2000 , caractérisés par une pression beaucoup moins forte sur les enseignants et des références moins marquées aux performances des écoles, ce qui permet probablement de comprendre pourquoi le rapport à ce type d'établissements y est vécu de manière différente.

Par ailleurs, indépendamment du leadership manifesté par la direction et du climat relationnel présent au sein de l'établissement d'enseignement, les conditions pratiques de travail influencent également la stabilité professionnelle de l'enseignant. Les ressources disponibles, tant financières que matérielles, constituent un facteur ayant un impact sur la volonté de maintien au sein de l'établissement. En effet, un équipement inadéquat pour dispenser un enseignement de qualité ou un accès insuffisant aux ressources jugées nécessaires peuvent mener le membre du personnel à ne pas se sentir pris en considération par la direction ou l'administration scolaire et à s'estimer moins compétent qu'il ne le souhaiterait. Toutefois, même aux États-Unis, rares sont les recherches ayant mesuré l'impact de l'équipement et des ressources mis à disposition sur la rétention du personnel (Ladd, 2011).

Enfin, la charge de travail imputée et la répartition des heures de cours conditionnent également les choix de carrière. Selon Luekens, Lyter et Fox (2004), elles sont à l'origine de $40 \%$ des demandes de changement d'affectation sur le territoire américain et constituent le premier motif de transfert entre établissements. Ainsi, les enseignants qui se voient confier des matières qui dépassent leur formation initiale, qui sont amenés à enseigner des cours multiples à différents niveaux d'éducation ou qui subissent des horaires inconfortables les astreignant à commencer tôt et à terminer tard tout en ayant plusieurs heures inoccupées au cours de la journée, seront davantage enclins à changer d'école voire à quitter la profession (Donaldson \& Johnson, 2010). Une recherche en cours menée en Belgique francophone (Delvaux, Desmarez, Dupriez et al., à paraître) attire également l'attention sur le poids majeur des conditions d'emploi face au risque d'abandon du métier par les enseignants novices. Trois caractéristiques liées à ces conditions d'emploi apparaissent ainsi comme fortement associées au risque de sortie précoce du métier : le nombre de mois de travail lors de la première année d'exercice, le pourcentage de temps de travail lors de cette même année et le moment du premier recrutement. II en ressort globalement que les enseignants ne disposant pas d'un emploi à temps plein, connaissant des périodes d'absence d'emploi entre deux périodes de travail ou ayant été recrutés plusieurs mois après le début de l'année scolaire sont de loin les plus susceptibles d'abandonner rapidement le métier.

\section{CONCLUSION}

La question de l'attrition professionnelle et de la migration des travailleurs entre des employeurs distincts est une préoccupation ancienne, abondamment explorée par la littérature scientifique, en particulier dans le domaine du management et de la 
gestion des ressources humaines. Cette littérature a permis de mettre en évidence la pluralité des facteurs individuels et organisationnels qui affectent l'intention de quitter un lieu de travail. Elle a notamment souligné le rôle médiateur joué par la satisfaction professionnelle et l'engagement organisationnel dans ce processus.

Dans le champ scolaire, les recherches les plus anciennes ont porté sur le phénomène d'attrition et avaient pour objectif d'identifier les facteurs tant individuels qu'organisationnels corrélés avec les risques de sortie du métier. Les travaux de nature économique ont également veillé à identifier le rôle des incitants (monétaires et autres) dans les décisions de réorientation professionnelle. Plus récemment, et parallèlement à l'évolution des politiques éducatives mettant au premier plan le rôle des établissements scolaires dans la régulation locale des systèmes éducatifs, c'est le concept de turnover qui s'est imposé dans la mesure où il résume, pour un établissement singulier, le taux de rotation de son personnel, qu'il s'agisse d'un changement d'établissement ou d'un abandon de la profession. À ce titre, un ensemble de facteurs, liés tant à l'enseignant qu'à l'organisation au sein de laquelle il est inséré, contribuent à la compréhension du phénomène de turnover.

D'un point de vue individuel, les enseignants novices présentent des taux de migration et d'attrition plus élevés que leurs collègues plus expérimentés. De part et d'autre de l'Atlantique, de nombreuses recherches ont mis en évidence que ces départs précoces - volontaires et involontaires - sont essentiellement consécutifs à une trajectoire professionnelle précaire ainsi qu'aux affectations fréquentes au sein d'établissements scolaires présentant des conditions de travail plus difficiles qui caractérisent le personnel débutant. De plus, la formation initiale du membre du personnel influence aussi sa stabilité professionnelle. La littérature anglo-saxonne et européenne a montré que les individus ne possédant pas les titres requis pour l'enseignement de la matière qui leur est attribuée ou ne disposant pas d'une formation pédagogique, sont plus enclins à quitter l'établissement scolaire au sein duquel ils exercent ou même à abandonner la profession. Ceux-ci sont en effet moins préparés aux exigences du métier d'enseignant. II apparaît également que les enseignants diplômés au terme d'une formation de longue durée sont les plus susceptibles de quitter le métier, probablement parce que leur diplôme est davantage « monnayable » sur d'autres marchés du travail. Cette tendance à quitter prématurément sa fonction est également plus marquée parmi les enseignants professant dans l'enseignement spécialisé nord-américain. Au sein de l'enseignement général, la littérature nord-américaine n'est pas unanime quant à l'influence de la discipline enseignée sur la stabilité du personnel. Si la majorité des auteurs soutiennent que les taux de turnover sont plus élevés parmi le personnel dispensant les cours de sciences, de mathématiques et dans une moindre mesure, de langue maternelle, d'autres, moins nombreux, ne mentionnent aucune différence statistiquement significative sur la base de la matière enseignée et tendent à montrer que les motifs de migration sont les mêmes pour les enseignants de mathématiques et de sciences que pour les enseignants d'autres disciplines (Ingersoll \& May, 2012). La littérature américaine souligne également l'importance de l'impact de l'origine ethnique du personnel et soutient que les enseignants issus de l'immigration sont plus stables que leurs collègues américains blancs. Par contre, en ce qui concerne l'influence du genre, tant en Europe qu'aux États-Unis, il n'existe pas d'assentiment généralisé au sein de la littérature. Ainsi, selon les caractéristiques individuelles et le contexte du travail, les auteurs mettent en exergue une stabilité professionnelle plus importante tantôt pour les enseignants et tantôt pour leurs collègues de sexe féminin.

Indépendamment de ces dimensions objectives, des facteurs subjectifs propres à l'individu tels que sa satisfaction professionnelle et l'engagement qu'il témoigne envers l'organisation ont également été considérés. Plutôt que d'étudier les déter- 
minants du turnover ou de l'abandon du métier, cette littérature s'intéresse aux conditions favorables à la satisfaction professionnelle et à l'engagement organisationnel, qui sont considérés comme des éléments favorables au maintien de l'individu dans la profession et dans l'organisation. De nombreux auteurs anglo-saxons et européens ont étudié l'impact d'un ensemble de facteurs tels que le genre et l'ancienneté sur chacune de ces deux variables. En ce qui concerne la satisfaction professionnelle, si les résultats des recherches mettent souvent en exergue une relation significative avec les différentes variables explicatives, la littérature n'est pas stabilisée en ce qui concerne l'intensité et l'orientation des relations observées. Quant à l'engagement organisationnel, l'étude de sa relation avec le genre conduit à des résultats contrastés dans la littérature. De nombreux auteurs s'accordent par contre sur la relation entre le niveau d'ancienneté et l'engagement envers l'organisation. Ce dernier est souvent plus manifeste parmi les membres du personnel ayant acquis quelques années d'ancienneté car les enseignants débutants tendent davantage à se focaliser sur les éléments directement liés au déroulement des tâches quotidiennes.

À l'échelle de l'établissement, les caractéristiques propres au public scolaire ainsi que celles liées à la dynamique organisationnelle ont un impact sur les taux de turnover. D'une part, la littérature met en exergue que la composition sociale, ethnique et académique de l'école influence la stabilité des enseignants qui y professent. Plus précisément, les taux de migration et d'attrition sont nettement plus élevés au sein des établissements accueillant un pourcentage important d'élèves socio-économiquement défavorisés, issus de minorités ethniques et/ou présentant des difficultés d'apprentissage. D'autre part, la littérature internationale montre que le leadership manifesté par la direction et le climat de l'école sont associés à la mobilité professionnelle des enseignants. Ainsi, la manifestation d'un leadership transformationnel valorisant l'autonomie du personnel et incitant les enseignants à participer aux prises de décisions tend à diminuer le turnover. La direction joue également un rôle positif quand elle peut témoigner d'un soutien aux enseignants confrontés à des problèmes pédagogiques ou de discipline. Outre la direction, les collègues et les élèves contribuent de manière significative à la stabilité professionnelle de l'enseignant. En effet, l'existence d'un climat collaboratif entre collègues ainsi que la présence d'un environnement respectueux et propice aux apprentissages des élèves concourent au maintien des enseignants dans l'établissement et, corollairement, au sein de la profession. Enfin, l'impact des conditions pragmatiques de travail n'est pas à négliger. Les ressources financières et matérielles disponibles ainsi que la charge de travail imputée et la répartition des heures de cours conditionnent aussi les choix de carrière et font partie, à ce titre, des motifs principaux de demandes de changements d'affectation.

Au-delà de cet ensemble de résultats, comme nous l'avons déjà souligné, la nature même des interrogations et des problématiques de recherche a largement évolué. Si le turnover des enseignants s'impose aujourd'hui comme un objet de recherche significatif, c'est entre autres parce que l'établissement scolaire est devenu dans de nombreux pays un niveau-clé d'implémentation des politiques éducatives. La question de la stabilisation des enseignants à cette échelle devient dès lors simultanément une question-clé de politique éducative et une question de recherche pertinente. On pourrait aussi relever - et certains des résultats présentés dans cette revue de la littérature l'attestent - la concomitance entre cette ligne de recherche et le développement de travaux autour du site-based management ou de la gestion locale des établissements scolaires. Dans une telle perspective, la capacité à attirer des enseignants et à les garder devient un indice d'un management de qualité. 
À cet égard, ce n'est sans doute pas une coïncidence de constater que cette question de la migration professionnelle des enseignants demeure aujourd'hui peu présente dans la recherche française en éducation. Nous l'expliquons par le fait que la France n'a accordé qu'assez tardivement et assez modérément de l'autonomie de gestion à ses établissements scolaires. Par ailleurs, le modèle français de recrutement et de gestion des carrières enseignantes demeure largement centralisé et l'autonomie des établissements sur ces questions est particulièrement modeste. Ces deux caractéristiques prennent par ailleurs racine et signification dans le modèle politique de l'école républicaine qui, par définition, considère les établissements comme des entités davantage semblables que singulières et les enseignants (ou les cadres) comme des agents de l'État plutôt que comme des acteurs identifiés à un lieu de travail spécifique. Dans un tel environnement institutionnel, la question de la migration professionnelle peut difficilement être pensée à l'échelle locale et relève davantage, quand elle émerge, de choix posés en matière de gestion régionale de la mobilité et de l'affectation des enseignants. Cette observation nous permet de revenir sur un des éléments que nous avions souligné dans l'introduction : cette revue de la littérature a la faiblesse de faire l'impasse, faute de matériaux disponibles, sur la relation entre des environnements politiques et institutionnels d'une part et la question du turnover d'autre part.

Si cette lacune s'explique par le fait que la plupart des recherches mentionnées ne concernent qu'un seul système éducatif, avec donc peu de variations à cet égard, c'est d'un point de vue théorique une lacune qu'il s'agira de combler. En effet, les questions de migration, de turnover et d'attrition des enseignants ne peuvent être réduites à des dimensions individuelles et organisationnelles. Plusieurs fois dans cette revue de la littérature, nous avons d'ailleurs eu tendance à expliquer la diversité des résultats présents dans la littérature par la diversité des environnements institutionnels où ont été menées les recherches mentionnées. En termes de politiques éducatives, un gisement de recherches particulièrement fécond devrait à nos yeux porter sur la diversité des modes de gestion des carrières enseignantes. Entre le traditionnel arrangement local qui caractérisait jusqu'il y a peu le système scolaire belge (Draelants, Dupriez \& Maroy, 2003) et l'entrée dans un statut de fonctionnaire d'État, il y a de multiples différences qui sans aucun doute vont affecter la stabilité de l'enseignant dans son métier et, quoique peut-être dans une moindre mesure, dans un lieu spécifique de travail. De telles recherches gagneraient à identifier les variations liées aux niveaux de prise de décision, mais aussi à la manière dont différents systèmes éducatifs ont pensé et administré des questions aussi diverses que le recrutement, la promotion, les conditions de rémunération ou encore l'accompagnement éventuel des premières années dans le métier.

Par rapport aux niveaux où se prend la décision (de recruter par exemple), il existe aujourd'hui dans le monde une grande variété de situations, et la recherche maîtrise au final très peu quelles sont les différences entre un modèle suédois (où tant le recrutement que le salaire se décident largement à une échelle locale) et le modèle français (où ces questions font l'objet d'une régulation nettement plus centralisée). Par rapport à d'autres dimensions de gestion des carrières, peu d'éléments ont fait l'objet d'une analyse systématique et la relation avec la double question de l'attrition et de la migration professionnelle demeure peu étudiée. Notons toutefois les travaux ayant porté sur les politiques d'accompagnement des enseignants débutants, lesquelles sont de plus en plus fréquentes dans les systèmes éducatifs des pays anglo-saxons (Smith \& Ingersoll, 2004). Plusieurs recherches ont montré que l'existence de programmes bien définis d'encadrement, d'accompagnement et de soutien diminue de manière significative les départs précoces de la profession. Ainsi, dans l'État du New Jersey (États-Unis), Gold (1999; cité par Holloway, 2002) 
met en évidence qu'alors que le taux d'attrition au cours de la première année est de $18 \%$ pour les enseignants ne bénéficiant d'aucune forme de soutien, il se situe aux alentours de $5 \%$ pour ceux ayant été encadrés. Notons également, dans un autre domaine, les travaux qui ont porté sur l'impact du niveau de salaire sur la stabilité professionnelle. Dans un pays comme les États-Unis où le salaire est défini en fonction du district, les chercheurs ont de longue date observé une migration vers les districts où les salaires sont plus élevés et des taux d'abandon de la profession plus importants dans les districts où les salaires sont plus faibles (Imazeki, 2005).

Mais au-delà des politiques spécifiques à la gestion des carrières, d'autres politiques (scolaires et non scolaires) mériteraient d'être prises en compte pour comprendre ce qui motive l'attitude des enseignants en termes de migration et d'attrition professionnelles. Mentionnons à cet égard, à titre d'exemple, les travaux de Clotfelter, Ladd, Vigdor et alii (2004) montrant que les politiques d'accountability et de pression sur les écoles développées dans certains États américains s'accompagnent d'un accroissement du turnover dans les écoles concentrant des élèves avec des difficultés d'apprentissage. De manière plus large, on pourrait également faire référence à d'autres types de politiques non spécifiques au champ scolaire mais qui sont susceptibles d'affecter la migration et l'attrition professionnelles. Comme nous l'avons mentionné dans le cadre de ce texte (Falch \& Strom, 2005), les politiques en matière de congé parental (et la manière dont elles favorisent le congé du père et/ou de la mère) sont parmi les plus susceptibles d'affecter les carrières professionnelles, notamment au sein du secteur de l'enseignement.

Mentionnons enfin, sur un registre différent, que la plupart des analyses et recherches présentées dans ce texte font assez largement abstraction du « contenu » du travail enseignant. Une recherche récente (Cochran-Smith, McQuillan, Mitchell et al., 2012) a ouvert la voie dans ce sens et mis en relation différentes facettes de la pratique enseignante, comme la gestion des relations sociales au sein de la classe, la planification du curriculum, le type de pratiques pédagogiques, les conceptions de l'apprentissage des élèves et de leur développement professionnel, avec les décisions de carrière d'enseignants durant leurs cinq premières années dans le métier. Cette étude a montré que les décisions de carrière, au-delà des conditions d'emploi, sont également liées à la qualité des pratiques d'enseignement développées, aux modalités de planification du curriculum, aux opportunités d'apprentissages qu'ils construisent pour leurs élèves, à la manière dont ils s'insèrent dans l'équipe pédagogique et apprennent de leurs pratiques. Pour élargir cette perspective de recherche, des recherches futures pourraient également envisager de s'appuyer sur les recherches développées autour des motivations et aspirations à devenir enseignant (en première ou deuxième carrière), de manière à traiter de façon plus approfondie les conceptions du métier et leurs inscriptions dans des mondes divers (Boltanski \& Thévenot, 1991) et la façon dont ces conceptions, plus ou moins en conflit avec les pratiques de classe et les pratiques/conceptions des collègues, jouent sur la rétention des enseignants dans l'établissement et dans le métier.

Sandrine Lothaire

Université catholique de Louvain (Belgique), GIRSEF sandrine.lothaire@uclouvain.be

Xavier Dumay

Université catholique de Louvain (Belgique), GIRSEF xavier.dumay@uclouvain.be

Vincent Dupriez

Université catholique de Louvain (Belgique), GIRSEF vincent.dupriez@uclouvain.be 


\section{NOTES}

1 Ce texte s'inscrit dans le cadre d'une recherche sur les marchés du travail des enseignants financée par le Fonds de la recherche scientifique (FRS-FNRS) à travers la convention FRFC $n^{\circ}$ 2.4579.11. La recherche, coordonnée par Vincent Dupriez (UCL), associe des chercheurs du GIRSEF (UCL) et de METICES (ULB).

2 Pour les références francophones, il s'agit de : "(déterminants du) turnover des employés", "(déterminants du) turnover enseignant", "(déterminants des) migrations des enseignants”, "(déterminants de) l'attrition des enseignants", “(déterminants de) l'intention de partir de l'enseignement”, "(déterminants de) la rétention des enseignants", "(déterminants de) l'intention de rester dans l'enseignement", "(déterminants de) la satisfaction professionnelle", "(déterminants de) l'engagement envers l'organisation", "(déterminants de) la satisfaction professionnelle des enseignants", "(déterminants de) l'engagement organisationnel des enseignants", "turnover enseignant et satisfaction professionnelle" et "turnover enseignant et engagement organisationnel". En ce qui concerne les références anglophones, elles ont été obtenues sur base des mots-clés suivants : "(determinants of) employee's turnover", “(determinants of) teacher's turnover", "(determinants of) teacher's migration", "(determinants of) teacher's attrition", "(determinants of) teacher's intention to leave", "(determinants of) teacher's retention", "(determinants of) teacher's intention to stay", "(determinants of) employee's job satisfaction", "(determinants of) employee's organizational commitment", "(determinants of) teacher's job satisfaction", "(determinants of) teacher's organizational commitment", "teacher's turnover and job satisfaction" et "teacher's turnover and organizational commitment".

3 Quel que soit le niveau d'enseignement.

4 Les données ne portent que sur les établissements d'enseignement secondaire ordinaire situés en Flandre.

5 En Communauté française de Belgique, en Allemagne et au Luxembourg, plus de $40 \%$ des élèves âgés de 15 ans sont scolarisés dans des établissements pour lesquels la direction déplore des difficultés à recruter et à stabiliser des enseignants qualifiés. Dans les deux autres communautés linguistiques belges, au PaysBas et au Royaume-Uni, le pourcentage est légèrement moins élevé et concerne entre 20 et $40 \%$ des élèves.

6 Les auteurs précisent que le turnover recouvre les problématiques d'attrition et de migration.

7 Dans le cadre de cette étude, le turnover est synonyme d'attrition.

8 Le turnover recouvre ici les problématiques des migrations et des attritions.

9 Dès lors, la somme des taux d'attrition et de migration annuels est égale à $12 \%$ pour l'enseignement ordinaire et à $20 \%$ pour l'enseignement spécialisé.

10 Le concept de turnover se rapporte tant aux migrations qu'aux attritions.

11 Les auteurs précisent qu'ils ne distinguent pas les enseignants qui quittent l'établissement de ceux qui quittent le district voire la profession. Le concept de turnover recouvre dès lors les migrations et les attritions.

12 L'administration des États-Unis répertorie une diversité de catégories raciales et ethniques présentes sur le territoire américain parmi lesquelles figurent les « américains blancs », les « afro-américains » et les « hispano-américains ".

13 Lycée au sein duquel les enfants d'ouvriers occupent une place supérieure à leur proportion nationale.

14 Lycée au sein duquel certaines catégories socioprofessionnelles des parents d'élèves (cadres supérieurs et professions libérales) sont massivement surreprésentées.

15 Dans le cadre de cet article, la notion de turnover est assimilée à celle d'attrition.

16 Le concept de turnover recouvre ici les problématiques de la migration et de l'attrition.

17 La mobilité enseignante inclut les notions de migration et d'attrition.

18 Le turnover se réfère aux migrations et aux attritions.

19 L'auteur s'intéresse à l'intention de quitter l'établissement d'enseignement. 


\section{BIBLIOGRAPHIE}

ADAMS G. J. (1996). "Using a Cox regression model to examine voluntary teacher turnover ». The Journal of Experimental Education, vol. 64, n० 3, p. 267-285.

ALLENSWORTH E., PONISCIAK S. \& MAZZEO C. (2009). The schools teachers leave: teacher mobility in Chicago Public Schools. Consortium on Chicago School Research at the University of Chicago Urban Education Institute.

ANDERSON J. C. \& MILKOVICH G. T. (1980). «Propensity to leave: a preliminary examination of March and Simon's model ". Industrial Relations, vol. 35, n॰ 2, p. 279294.

BARRÈRE A. \& MARTUCCELLI D. (1996). “L'école à l'épreuve de l'ethnicité ». Les Annales de la recherche urbaine, n० 75, p. 51-58.

BISHAY A. (1996). "Teacher motivation and job satisfaction: a study employing the experience sampling method ". Journal of Undergraduate Sciences, n 3, p. 147-154.

BLASE J. J. (1986). «A qualitative analysis of sources of teacher stress: consequences for performance ». American Educational Research Journal, vol. 23, no 1, p. 13-40.

BLAU G. J. \& BOAL K. B. (1987). «Conceptualizing how job involvement and organizational commitment affect turnover and absenteeism ». Academy of Management Review, vol. $12, n^{\circ} 2$, p. 288-300.

BOE E. E, BOBBITT S. A., COOK L. H., BARKANIC G. \& MAISLIN G. (1998). Teacher turnover in eight cognate areas: national trends and predictors. University of Pennsylvania : Department of Education.

BOE E. E, BOBBITT S. A., COOK L. H., WHITENER S. D. \& WEBER A. L. (1997). « Why didst thou go? Predictors of retention, transfer and attrition of special and general education teachers from a national perspective ". The Journal of Special Education, vol. 30, n० 4, p. 390-411.

BOGLER R. \& SOMECH A. (2004). "Influence of teacher empowerment on teachers" organizational commitment, professional commitment and organizational citizenship behavior in schools ". Teaching and Teacher Education, vol. 20, n 3, p. 277-289.

BOLTANSKI L. \& THÉVENOT L. (1991). De la justification : les économies de la grandeur. Paris : Gallimard.

BONESRONNING H., FALCH T. \& STROM B. (2005). "Teacher sorting, teacher quality and student composition ». European Economic Review, no 49, p. 457-483.

BOSWELL W. R., SHIPP A. J., PAYNE S. C. \& CULBERTSON S. S. (2009). "Changes in newcomer job satisfaction over time: examining the pattern of honeymoons and hangovers ». Journal of Applied Psychology, vol. 94, no 4, p. 844-858.

BOYD D., GROSSMAN P., ING M., LANKFORD H., LOEB S. \& WYCKOFF J. (2011). "The influence of school administrators on teacher retention decisions ". American Educational Research Journal, vol. 48, n०2, p. 303-333.

CHA S. \& COHEN-VOGEL L. (2011). "Why they quit: a focused look at teachers who leave for other occupations ". School Effectiveness \& School Improvement, vol. 22, n० 4, p. 371-392.

CHAPMAN D. W. (1983). "A model of the influences on teacher retention ". Journal of Teacher Education, vol. 34, n० 5, p. 43-49.

CHARTERS W. W. (1970). "Some factors affecting teacher survival rates in school districts ». American Educational Research Journal, vol. 7, no 1, p. 1-27.

CLOTFELTER C. T., LADD H. F., VIGDOR J. L. \& DIAZ R. A. (2004). « Do school accountability systems make it more difficult for low performing schools to attract and retain high quality teachers? ». Journal of Policy Analysis and Management, n० 23, p. 251-271.

COLADARCI T. (1992). "Teachers' sense of efficacy and commitment to teaching ». Journal of Experimental Education, vol. 60, no 4, p. 323-337.

CONLEY S. \& YOU S. (2009). «Teacher role stress, satisfaction, commitment, and intentions to leave: a structural model ». Psychological reports, vol. 105, n० 3, p. 771-786.

COCHRAN-SMITH M., McQUILLAN P., MITCHELL K. et al. (2012). " A longitudinal study of teaching practice and early career decisions: a cautionary tale ". American Educational Research Journal, vol. 49, n० 6, p. 1-37.

DELVAUX B., DESMAREZ P., DUPRIEZ V., LOTHAIRE S. \& VEINSTEIN M. (à paraître). « Les enseignants novices : analyse des conditions d'entrée dans le métier, des trajectoires professionnelles et des sorties du métier ». Les Cahiers de recherche du Girsef. 
DEMAILLY L. (1991). Le collège. Crise, mythes et métiers. Lille: Presses universitaires de Lille.

DEVOS C. (2012). The role of self-aspect importance and social working environment on beginning teachers' self-efficacy and feelings of depression. Thèse de doctorat, sciences psychologiques et de l'éducation, université catholique de Louvain (Belgique).

DEVOS C., DUPRIEZ V. \& PAQUAY L. (2012). " Does the social working environment predict beginning teachers' self efficacy and feelings of depression? ". Teaching and Teacher Education, vol. 28, no 2, p. 206-217.

DOLTON P. \& van der KLAAUW W. (1999). « The turnover of teachers: a competing risks explanation ". The Review of Economics and Statistics, vol. 81, n० 3, p. 543-552.

DONALDSON M. L. \& JOHNSON S. M. (2010). " The price of misassignment: the role of teaching assignments in teach for America teachers' exit from low-income schools and the teaching profession ". Educational Evaluation and Policy Analysis, vol. 32, n० 2, p. 299-323.

DRAELANTS H., DUPRIEZ V. \& MAROY C. (2003). « Le système scolaire en Communauté française ». Dossiers du Crisp, n० 59, p. 3-116.

DUBET F. (2002). Le déclin de l'institution. Paris : Éd. du Seuil.

DUMAY X. \& GALAND B. (2012). «The multilevel impact of transformational leadership on teacher commitment: cognitive and motivational pathways ". British Educational Research Journal, vol. 38, no 5, p. 703-729.

EUROPEAN COMMISSION (2012). Key data on education in Europe 2012.

EVANS E. D. \& TRIBBLE M. (1986). "Perceived teaching problems, self-efficacy and commitment to teaching among preservice teachers ". The Journal of Educational Research, vol. 80, n², p. 81-85.

EVETTS J. (1988). "Returning to teaching: the career breaks and returns of married women primary headteachers ». British Journal of Sociology of Education, vol. 9, n० 1, p. 81-96.

FALCH T. \& STROM B. (2005). "Teacher turnover and non-pecuniary factors ». Economics of Education Review, no 24, p. 611-631.

FARKAS A. J. \& TETRICK L. E. (1989). «A Three-Wave Longitudinal Analysis of the Causal Ordering of Satisfaction and Commitment on Turnover Decisions ». Journal of Applied Psychology, vol. 74, n 6, p. 855-868.

FESTINGER L. (1957). A theory of cognitive dissonance. Evanston (États-Unis) : Row, Peterson.

GOLD Y. (1999). "Beginning teacher support ». In J. Sikula, T. Buttery \& E. Guyton, Handbook of research in teacher education. New York: Macmillan, p. 548-594.

GREENBERG D. \& McCALL J. (1974). «Teacher mobility and allocation ». The Journal of Human Resources, vol. 9, no 4, p. 480-502.

GRISSMER D. W. \& KIRBY S. N. (1992). Patterns of attrition among Indiana teachers, 1965-1987: an executive summary. RAND.

GUILLEMETTE F. (2006). L'engagement des enseignants du primaire et du secondaire dans leur développement professionnel. Thèse de doctorat, sciences de l'éducation, université du Québec à Trois-Rivières en association avec l'université du Québec à Montréal.

HANUSHEK E. A., KAIN J. F. \& RIVKIN S. G. (2004). "Why public schools lose teachers ". Journal of Human Resources, vol. 39, n० 2, p. 326-354.

HART A. W. \& MURPHY M. J. (1990). «New teachers react to redesigned teacher work ». American Journal of Education, vol. 98, n० 3, p. 224-250.

HOLLOWAY J. H. (2001). "The benefits of mentoring ». Educational Leadership, vol. 58, n० 8, p. 85-86.

HOLTOM B. C., MITCHELL T. R., LEE T. W. \& EBERLY M. B. (2008). « Turnover and retention research: A glance at the past, a closer review of the present, and a venture into the future ". The Academy of Management Annals, vol. 2, no 1, p. 231-274.

HUBERMAN M. (1993). The lives of teachers. New York: Teachers College Press.

HUMPHREY S. E., NAHRGANG J. D. \& MORGESON F. P. (2007). «Integrating motivational, social, and contextual work design features: a meta-analytic summary and theoretical extension of the work design literature ". Journal of Applied Psychology, vol. 92, n 5, p. $1332-1356$. 
IMAZEKI J. (2005). "Teacher salaries and teacher attrition ». Economics of Education Review, n०24, p. 431-449.

INGERSOLL R. M. (2000). Turnover among mathematics and science teachers in the U.S. University of Pennsylvania, Graduate school of education, Prepared for the National Commission on Mathematics and Science Teaching for the 21st century.

INGERSOLL R. M. (2001a). "Teacher turnover and teacher shortages: an organizational analysis ». American Educational Research Journal, vol. 38, n 3, p. 499-534.

INGERSOLL R. M. (2001b). Teacher turnover, teacher shortages and the organization of schools. University of Washington, Center for the Study of Teaching and Policy.

INGERSOLL R. M. (2002). " The teacher shortage: a case of wrong diagnosis and wrong prescription ". NASSP Bulletin, vol. 86, n 631, p. 16-31.

INGERSOLL R. M. \& MAY H. (2012). " The magnitude, destinations, and determinants of mathematics and science teacher turnover ". Educational Evaluation and Policy Analysis, vol. 34, no 4, p. 464-485.

JACKSON C. K. (2009). "Student demographics, teacher sorting and teacher quality: evidence from the end of school desegregation ". Journal of Labor Economics, vol. 27, n० 2, p. 213-256.

JOHNSON S. M. (2006). The workplace matters: Teacher quality, retention and effectiveness. Washington : National Education Association.

JOHNSON S. M., BERG J. H. \& DONALDSON M. L. (2005). Who stays in teaching and why: a review of the literature on teacher retention. Washington : NRTA.

KIRBY S. N., BERENDS M. \& NAFTEL S. (1999). "Supply and demand of minority teachers in Texas: problems and prospects ". Educational Evaluation and Policy Analysis, vol. 21, no 1, p. 47-66.

KLASSEN R. M. \& CHIU M. M. (2010). " Effects on teachers' self-efficacy and job satisfaction: teacher gender, years of experience and job stress ". Journal of Educational Psychology, vol. 102, n 3, p. 741-756.

KUKLA-ACEVEDO S. (2009). "Leavers, movers, and stayers: the role of workplace conditions in teacher mobility decisions ". The Journal of Educational Research, vol. 102, n० 6, p. 443-452.

LADD H. F. (2011). " Teachers' perceptions of their working conditions: how predictive of planned and actual teacher movement ". Educational Evaluation and Policy Analysis, vol. 33, no2, p. 235-261.

LANKFORD H., LOEB S. \& WYCKOFF J. (2002). « Teacher sorting and the plight of urban schools: a descriptive analysis ". Educational Evaluation and Policy Analysis, vol. 24, no 1, p. 37-62.

LAWLER E. E. \& PORTER L. W. (1967). «The effect of performance on job satisfaction ». Industrial Relation, A Journal of Economy and Society, vol. 7, no 1, p. 20-28.

LÉGER A. (1981). "Les déterminants sociaux des carrières enseignantes ». Revue française de sociologie, vol. 22 , no 4, p. 549-574.

LÉGER A. (1983). Enseignants du secondaire. Paris : PUF.

LEITHWOOD K. A. \& POPLIN M. S. (1992). «The move toward transformational leadership ». Educational Leadership, vol. 49, no 5, p. 8-12.

LEVIN H. M. (1985). "Solving the shortage of mathematics and science teachers ". Educational Evaluation and Policy Analysis, vol. 7, no 4, p. 371-382.

LIU X. S. \& RAMSEY J. (2008). «Teachers' job satisfaction: analyses of the teacher followup survey in the United States for 2000-2001 ». Teaching and Teacher Education, n० 24, p. 1173-1184.

LOCKE E. A. (1969). «What is job satisfaction? ». Organizational Behavior and Human Performance, no 4, p. 309-336.

LOEB S., DARLING-HAMMOND L. \& LUCZAK J. (2005). " How teaching conditions predict teacher turnover in California schools". Peabody Journal of Education, vol. 80, n० 3 , p. 44-70.

LORTIE D. C. (1975). Schoolteacher: a sociological study. Chicago : University of Chicago Press.

LUEKENS M. T., LYTER D. M. \& FOX E. E. (2004). Teacher Attrition and Mobility: Results from the Teacher Follow-up Survey 2000-01 (NCES 2004-301). Washington: Department of Education, National Center for Education Statistics, U.S. Government Printing Office. 
MA X. \& MacMILLAN R. B. (1999). «Influences of workplace conditions on teachers' job satisfaction ". The journal of educational research, vol. 93, no 1, p. 39-47.

MALET R. (2011). «Un analyseur des formes de redéfinition stratégiques de l'État. Les politiques d'imputabilité en direction des enseignants en Angleterre et aux ÉtatsUnis ". Education comparée, no 5, p. 35-60.

MARCH G. L. \& SIMON H. A. (1958). Organizations. New York : Wiley.

MARK J. \& ANDERSON B. (1978). «Teacher survival rates: A current look ». American Educational Research Journal, vol. 15, n 3, p. 379-382.

MAROY C. \& CATTONAR B. (2002). «Pénurie et malaise enseignant ». La Revue nouvelle, vol. $115, n^{\circ} 12$, p. 44-62.

MENON M. E. \& CHRISTOU C. (2002). «Perceptions of future and current teachers on the organization of elementary schools: a dissonance approach to the investigation of job satisfaction ». Educational Research, vol. 44, no 1, p. 97-110.

MEURET D. (2007). Gouverner l'école. Paris : PUF.

MOWDAY R. T., STEERS R. M. \& PORTER L. W. (1979). « The measurement of organizational commitment ». Journal of Vocational Behavior, no 14, p. 224-247.

MUKAMURERA J. (1999). "Les trajectoires d'insertion des jeunes profs au Québec ». Vie pédagogique, no 111.

MURNANE R. J. \& OLSEN R. J. (1989). "The effect of salaries and opportunity costs on duration in teaching: evidence from Michigan ". Review of Economics and Statistics, vol. 71, no 2, p. 347-352.

MURNANE R. J., SINGER J. D. \& WILLETT J. B. (1987). "Changes in teacher salaries during the 1970s: the role of school district demographics ». Economics of Education Review, vol. 6, n० 4, p. 379-388.

NEWTON X. A., RIVERO R., FULLER B. \& DAUTER L. [s.d.]. Teacher stability and turnover in Los Angeles: the influence of teacher and school characteristics. Working Paper, Los Angeles Infrastructure Project, Policy analysis for California Education.

OCDE (2001). Regards sur l'éducation : les indicateurs de l'OCDE. Paris : OCDE.

PÉRIER P. (2003). Le métier d'enseignant dans les collèges et lycées au début des années 2000. Vanves: Les Dossiers, Ministère de l'Éducation nationale-DEP.

POCHARD M. (2008). Livre vert sur l'évolution du métier d'enseignant. En ligne : http:// www.education.gouv.fr/cid60320/commission-sur-le-metier-d-enseignant.html (consulté le 4 février 2013).

RAYOU P. \& van ZANTEN A. (2004). Enquête sur les nouveaux enseignants : changeront-ils l'école ? Paris: Bayard.

REES D. I. (1991). " Grievance procedure strength and teacher quits ". Industrial and Labor Relations Review, vol. 45, p. 31-43.

ROSENHOLTZ S. J. \& SIMPSON C. (1990). "Work conditions and the rise and fall of teachers' commitment ». Sociology of Education, vol. 63, no 4, p. 241-257.

RUMBERGER R. W. (1987). "The impact of salary differentials on teacher shortages and turnover: the case of mathematics and science teachers ". Economics of Education Review, vol. 6, no 4, p. 389-399.

SCAFIDI B., SJOQUIST D. L. \& STINEBRICKNER T. R. (2007). « Race, poverty and teacher mobility ". Economics of Education Review, vol. 26, n० 4, p. 145-149.

SCHLECHTY P. C. \& VANCE V. S. (1981). "Do academically able teachers leave education? The North Carolina case ». The Phi Delta Kappan, vol. 63, no 2, p. 106-112.

SHEN J. (1997). «Teacher retention and attrition in public school: evidence from SASS91". The Journal of Educational Research, vol. 91, n² 2, p. 81-88.

SHORT P. M., GREER J. T. \& MELVIN W. M. (1994). "Creating empowered schools: lessons in change ». Journal of Educational Research, vol. 32, n० 4, p. 38-52.

SMITH T. M. \& INGERSOLL R. M. (2004). «What are the effects of induction and mentoring on beginning teacher turnover? ". American Educational Research Journal, vol. 41, no 3, p. 681-714.

STRUNK K. O. \& ROBINSON J. P. (2006). " Oh, won't you stay: a multilevel analysis of the difficulties of retaining qualified teachers ». Peabody Journal of Education, vol. 81, n० 4, p. 65-94. 
TAYLOR D. L. \& TASHAKKORI A. (1995). "Decision participation and school climate as predictors of job satisfaction and teachers' sense of efficacy ». The Journal of Experimental Education, vol. 63, n 3, p. 217-230.

THEOBALD N. D. (1990). "An examination of the influence of personal, professional, and school district characteristics on public school teacher retention ". Economics of Education Review, vol. 9, no 3, p. 241-250.

TYREE A. K. (1996). "Conceptualizing and measuring commitment to high school teaching ». The Journal of Educational Research, vol. 89, n० 5, p. 295-304.

VANDENBERGHE V. (2000). "Leaving teaching in the French-speaking Community of Belgium: a duration analysis ». Education Economics, vol. 8, n० 3, p. 221-239.

van ZANTEN A. \& GROSPIRON M.-F. (2001). " Les carrières enseignantes dans les établissements difficiles : fuite, adaptation et développement professionnel ». VEI Enjeux, no 124, p. 224-268.

VENKATARAMANI V., GREEN S. G. \& SCHLEICHER D. J. (2010). «Well-connected leaders: the impact of leaders' social network ties on LMX and members' work attitudes ". Journal of Applied Psychology, vol. 95, n॰ 6, p. 1071-1084.

VLAAMS MINISTERIE VAN ONDERWIJS EN VORMING (2009). Arbeidsmarktrapport. Basisonderwijs en Secundair Onderwijs. En ligne : http://www.ond.vlaanderen.be/ beleid/personeel/files/AMR-2009.pdf (consulté le 23 mars 2012).

WEISS E. M. (1999). "Perceived workplace conditions and first-year teachers' morale, career choice commitment, and planned retention: a secondary analysis ". Teaching and Teacher Education, vol. 15, n० 8, p. 861- 879.

WELLER I., HOLTOM B. C., MATIASKE W. \& MELLEWIGT T. (2009). « Level and time effects of recruitment sources on early voluntary turnover ». Journal of Applied Psychology, vol. 94, no 5, p. 1146-1162.

WISNIEWSKI W. (1990). "The job satisfaction of teachers in Poland ". Comparative Education, vol. 26, no 2/3, p. 299-306. 